MATEC Web of Conferences 52, 01001 (2016)

DOI: $10.1051 /$ matecconf/20165201001

(C) Owned by the authors, published by EDP Sciences, 2016

\title{
Aircraft Performance Analysis in Conceptual Design Phase based on System-of-Systems Simulations
}

\author{
Yifeng Tian ${ }^{1, a}$, Hu Liu ${ }^{1}$ and Jun Huang ${ }^{1}$ \\ ${ }^{1}$ School of Aeronautic Science and Engineering, Beihang University, Beijing, China
}

\begin{abstract}
To obtain a competitive design in aircraft conceptual design phase, this paper propose an aircraft performance analysis method based on system-of-systems (SoS) simulations. The analysis process includes design space exploration and mission effectiveness analysis and the stochastic nature in SoS mission is captured based on a Monte Carlo method. Meanwhile as an example, the performance of UAVs which are applied in earthquake search and rescue SoS is analysed based on SoS simulations. The results show that the level of aircraft performance which leads to an optimum SoS effectiveness could be designed below the maximum performance. And it is necessary to apply SoS simulations into aircraft conceptual design phase to help designers optimize aircraft performance.
\end{abstract}

\section{Introduction}

In the past, the top-down decisions about aircraft performance parameters in conceptual design phase are usually made by chief engineers based on their experience, intuition and judgment [1]. And the value of original target design parameters are often set according to the existing aircraft or systems. To some degree, the design space exploration, parameter optimization and life-cycle cost are neglected, often leading to substantially unnecessary time and cost [2, 3]. Meanwhile, as the complexity in the fields of civil and military aviation is continuously increasing, aircraft usually carry out large system-of-systems (SoS) missions which make aircraft performance design more complicated due to the competition of different systems. So performance parameters should be analysed in depth.

The concept of SoS was first proposed in 1956 [4], but it was not until 1989 that SoS was introduced to engineering application [5]. It are usually made up of a multitude of independent but interactive systems. In recent years, this field has been growing significantly and SoS theory has been applied in many aeronautical engineering problems, such as air strike [6,7], civilian transportation [8, 9] and so on. Different methods have also been applied to deal with these SoS problems, such as surrogate models [6,7], mixed integer nonlinear programming [8], network theory [9], Agent [10], etc. Although there is still a lack of a common definition in the primary stage of SoS theory [10], the characteristics proposed by Maier [11] have been widely recognized, i.e., emergent behaviour, evolutionary development, operational independence of the elements, managerial independence of the elements and geographic distribution. The first two characteristics could also be found in systems because systems are established to acquire new capacity. However, the latter three characteristics are

\footnotetext{
${ }^{a}$ Corresponding author: tianyifeng_buaa@163.com
} 
rather unique. Because component systems in different geographic positions have a high autonomy but also interact with each other, the uncertainty and discontinuities increase the difficulties in analysing and predicting the results of SoS mission. So when aircraft carry out a SoS mission, mission effectiveness is not only affected by aircraft performance but also by other component systems. Due to the competition of different systems in an SoS, the aircraft with a low level of performance parameters may also lead to a high mission effectiveness. Thus the desired aircraft is not the one with maximum performance but the one which leads to an optimal mission effectiveness.

Although in conceptual design phase there is no existing aircraft to conduct experiments to study the relationship between aircraft performance and mission effectiveness, modelling and simulation in a computer provides a feasible way to creating the mapping from measure of performance to measure of effectiveness. Through establishing SoS model and operating simulation program repeatedly based on Monte Carlo simulations, large amount of data in the uncertainty environment could be obtained. These data can provide designers two aspects of assessment: exploring design space according to mission effectiveness and analysing mission effectiveness of different designs.

In order to illuminate the issue, an example about unmanned aerial vehicle (UAV) search and rescue $\mathrm{SoS}$ in earthquake relief is discussed in the following sections. The SoS model is built according to earthquake relief in the reality. Based on SoS simulations, the UAV performance is analysed from the perspective of both design space exploration and mission effectiveness analysis.

\section{Modelling process of UAV search and rescue SoS}

\subsection{Operation logic of mission simulation}

When establishing an SoS model, the operation logic of each component system and the interactive logic of different component systems are very important. In fact, the example UAV search and rescue SoS in earthquake relief is very complex. However, the purpose of this paper is mainly to illuminate the important role of SoS simulation in aircraft conceptual design phase, some reasonable simplifications are set to reduce the difficulty in modelling process. When establishing the models of component systems, UAVs, survivors, temporary base and helicopters are selected, regardless of ground rescue because the terrain is set as mountainous area and the mission time is limited. Meanwhile the weather system is not taken into account. However, if necessary, a more comprehensive SoS model could be built for mission simulations. Considering the autonomy of component systems, systems are modelled as Agent.
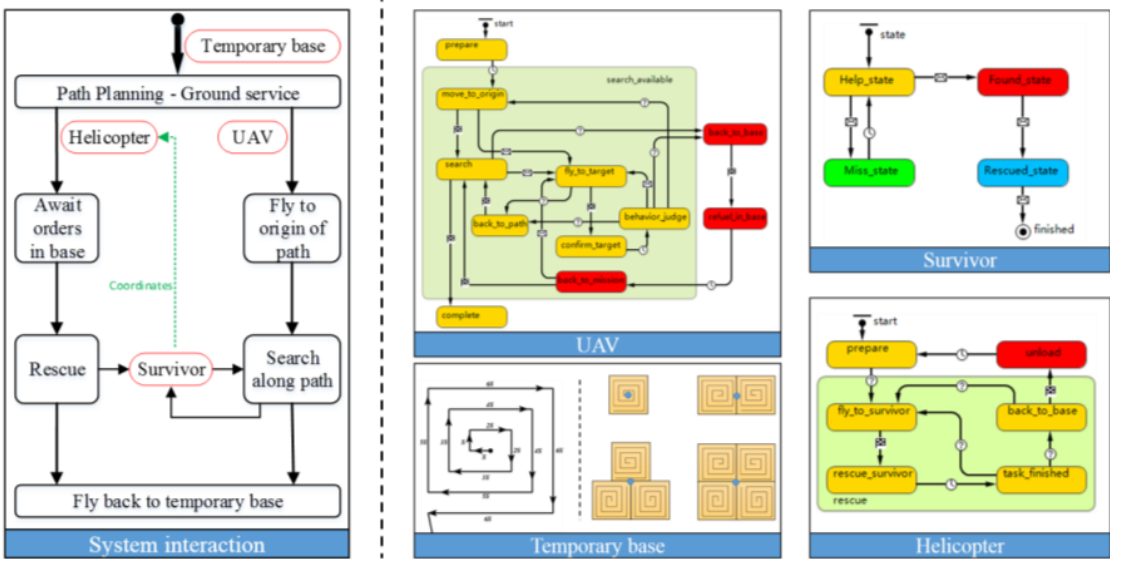

Figure 1. Operation logic of SoS mission.

The operation logic are shown as Fig.1. In the initial condition, survivors are distributed in the search area randomly. The temporary base plans paths based on the number of UAVs and mission area. UAVs carry out their search mission along the search path which is set as an expanding square 
pattern [12]. When a UAV finds a survivor, it will confirm the coordinates and send message to helicopters. Then a helicopter will fly to target to rescue the survivor. When UAVs finish searching mission area and survivors that are found have been rescued, the mission is finished. Each system has different operation states in its operation logic and systems change states according to active time, environment, coordinates, messages and etc. All logic must be validated through running and debugging the program repeatedly to conform the search and rescue logic in real-life scenario.

\subsection{Equations of flight mechanics for UAVs}

Without geometric design in early conceptual design phase, it is hard to get some aerodynamics data of UAVs. However, in SoS simulations UAVs operate based on the basic equations of flight mechanics, which provides a bridge from UAV performance to mission effectiveness. That is to say, different original design parameters lead to different UAV performance and different UAV performance lead to different mission effectiveness.

If a UAV is flying at a cruise speed of $V$, in every moment the motion state of UAVs could be regarded as a steady motion because the total weight or the fuel weight decreases very slowly. Thus the thrust $(T)$ equals the drag $(D)$ and the lift $(L)$ equals the total weight $(W)$. The relationship of the engine thrust $\left(T_{a}\right)$, lift-drag ratio $(K)$, and thrust efficiency $(\eta)$ can be calculated as Eq. 1 . The relationship of $W$, fuel consumption $\left(Q_{f}\right)$, thrust specific fuel consumption $\left(c_{f}\right)$ and time $(t)$ can be calculated as Eq. 2. The endurance $\left(T_{e}\right)$ and range $(R)$ could be calculated as Eq. 3 and Eq. 4 through the integral from the initial weight $\left(W_{l}\right)$ to the final weight $\left(W_{2}\right)$.

$$
\begin{gathered}
T=\eta T_{a}=\frac{W \times D}{L}=\frac{W}{K} \\
d t=\frac{d Q_{f}}{c_{f} T_{a}}=-\frac{d m}{c_{f} T_{a}}=-\frac{K \eta d W}{g c_{f} W} \\
T_{e}=-\int_{W_{1}}^{W_{2}} \frac{d W}{g c_{f} T_{a}}=\int_{W_{2}}^{W_{1}} \frac{K \eta}{g c_{f} W} d W \\
R=-\int_{W_{1}}^{W_{2}} \frac{V d W}{g c_{f} T_{a}}=\int_{W_{2}}^{W_{1}} \frac{V K \eta}{g c_{f} W} d W
\end{gathered}
$$

From the equations, it can be seen that some performance parameters could be calculated from other parameters, such as $T_{e}$ and $R$. So in this simulations, $K, W, T / W, c_{f}, \eta$ and $Q_{f}$ are selected as main design parameters to analyse the UAV performance. However, more design parameters could be added in simulations and analysed in depth as the research continues

\section{Analysis Process of SoS simulations}

SoS simulations have a significant reference value for aircraft design through exploring design space and analysing mission effectiveness. When operating simulations, the uncertainty must be taken into account. Thus a Monte Carlo simulation is used to capture the stochastic nature in the SoS mission.

\subsection{Design space exploration of performance parameters}

Before exploring design space, the design target should be decided clearly: a small size, light, portable UAV with a small size piston engine is needed in the earthquake relief. Although designers cannot predict the desirable design point in advance, the bounds of design space could be set based on technical experience or existing UAVs. The bounds should be set wide enough to cover possible design points. The value of parameters are set as a uniform distribution within the bounds to seek possible solution. The value are shown as Table 1.The main structural materials are usually wood, 
carbon fiber and fiberglass which can make UAVs lighter. So the value of $W_{\text {gtow }}$ could be set a little small, such as 10 to $15 \mathrm{~kg}$. The thrust of a small size engine is usually low so $T / W$ could be set as 0.4 to 0.8 . Compared with low $T / W$, a high $K$ is needed, such as 12 to $24 . c_{f}$ and $\eta$ could be set as 0.8 to $1.2 \mathrm{~kg} /(\mathrm{kg} \cdot \mathrm{h})$ and 0.7 to 0.9 respectively based on some engine characteristics. And then the space for fuel tank is usually not very large, so $W_{\text {fuel }}$ is set as 0.6 to $1.4 \mathrm{~kg}$. Designers could change the bounds according to a certain mission.

Table 1. List of Performance Parameters.

\begin{tabular}{|c|c|c|}
\hline Variable & Description & Distribution \\
\hline$W_{\text {gtow }}$ & Gross take-off weight $[\mathrm{kg}]$ & uniform $(10,15)$ \\
\hline$K$ & Lift-drag ratio & uniform $(12,24)$ \\
\hline$T / W$ & Thrust-weight ratio & uniform $(0.4,0.8)$ \\
\hline$c_{f}$ & Thrust specific fuel consumption $[\mathrm{kg} /(\mathrm{kg} \cdot \mathrm{h})]$ & uniform $(0.8,1.2)$ \\
\hline$\eta$ & Thrust efficiency & uniform $(0.7,0.9)$ \\
\hline$W_{\text {fuel }}$ & Fuel weight $[\mathrm{kg}]$ & uniform $(0.6,1.4)$ \\
\hline
\end{tabular}

The simulation scenario is shown as Fig. 2. The green house represents the temporary base. The pink circle represents a helicopter. The light blue circle represents a UAV and the area it has searched is shown as red shadows. The yellow circle represents survivors. They will change their initial coordinates in each run. If found by a UAV, their color changes to red and if rescued by a helicopter, its color changes to blue. In this scenario, an assumption is set that the UAV never miss a survivor. When operating the simulation based on a Monte Carlo, all other mission inputs keep the same except the performance parameters in design space.

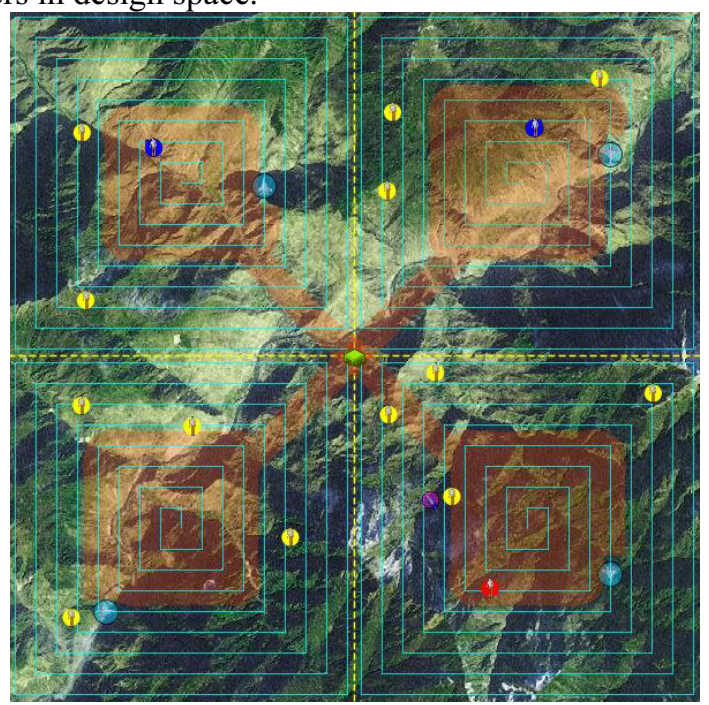

Figure 2. Simulation scenario of SoS mission.

In order to capture the stochastic nature, 30000 design points are used in a Monte Carlo. This amount is accuracy enough to populate the design space after a comparison experiment with 20000 and 40000 design points. In each run, mission time is chosen as the main effectiveness. The data shows that mission time of 30000 cases are all less than 600 minutes. However, the target is to design a competitive UAV and designers are more interested in decreasing the performance parameter without losing much mission effectiveness from the perspective of saving life-cycle cost, too short or too long mission time is not suitable. So the time interval is set as 120 minutes to 180 minutes and the 
number of cases in this interval is 20656 . The histogram and some statistics data are shown as Fig. 3. The histogram in yellow represents the selected cases.

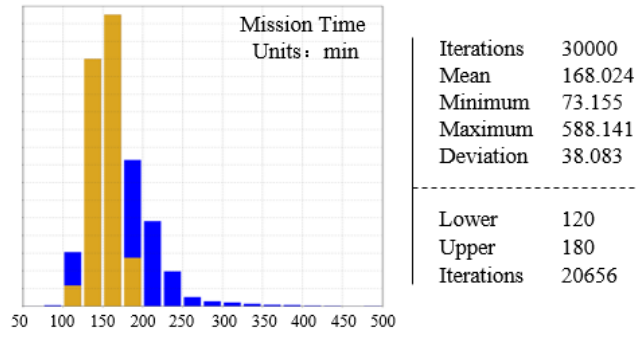

Figure 3. Histogram of All cases and selected cases.

Fig. 3 is not enough to reflect the relationship between performance parameters and mission effectiveness, the data analysis for design parameters should be researched in depth. Here choose $L / D$ and $T / W$ as an example to illuminate the issue, see Fig. 4. The $x$ axis show the interval of parameters. The histogram in light blue represents the distribution of all 30000 cases. The distribution of all cases is uniform because the distribution of the value of design parameters is uniform. The histogram in blue represents the distribution of selected cases. Fig. 4 (a) shows the relationship between $K$ and mission effectiveness. It can be seen that the height of blue bars increases sharply when $K$ changes from 12 to 18. And then it becomes steady. It is because increasing $K$ will decrease the thrust required, which can reduce fuel consumption and improve the endurance and range. The appropriate value could be set as 19 to 20, because it doesn't improve mission effectiveness further with a higher $K$. However, from Fig. 4 (b), it can be seen that the height of blue bars keeps relatively steady in the whole value interval. It is because with a relatively light total weight and a relatively high $K, T / W$ is not required too high. The appropriate value could be set as 0.4 or 0.5 . These optimal parameters will have an important reference value for the following design phases.

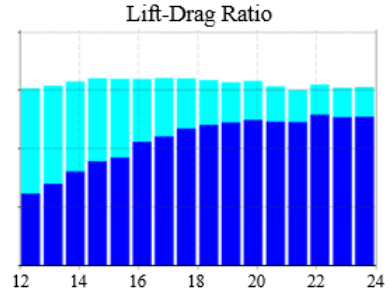

(a). Distribution of $K$.

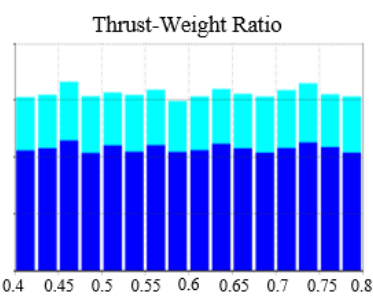

(b). Distribution of $T / W$.

Figure. 4. Histograms of cases from the view of parameters.

\subsection{Mission effectiveness analysis of performance designs}

If two designs are alternative to carry out a mission, SoS simulations can help designers select the more suitable one from the perspective of overall SoS optimum. The performance parameters are shown as Table 2. When comparing two different UAV designs, another parameter, detection probability $\left(P_{\text {lim }}\right)$ should be set. It is possible that the UAV with a higher speed may miss more survivors than the one with a lower speed. However, the former one could finish mission faster. So, it is hard to distinguish which one is better in advance before mission simulations. Here mission completeness $\left(D_{m c}\right)$ and time utilization ratio $\left(P_{t u}\right)$ is set as mission effectiveness, see Eq. 5 and Eq. 6 . $D_{m c}$ equals the number of the survivors that are rescued $\left(N_{\text {res }}\right)$ over the number of all survivors $\left(N_{s u r}\right)$. $P_{t u}$ equals $N_{r e s}$ over mission time $\left(T_{f i n}\right)$. Due to the uncertainty, the mission effectiveness will still change in each run even with the same UAV. Thus a Monte Carlo simulation is also used to capture the stochastic nature. For each UAV, the mission simulation will repeat 2000 times. 


$$
\begin{gathered}
D_{m c}=\frac{N_{r e s}}{N_{\text {sur }}} \\
P_{t u}=\frac{N_{r e s}}{T_{f i n}}
\end{gathered}
$$

Table 2. Performance parameters of two UAV designs.

\begin{tabular}{|c|c|c|c|}
\hline Variable & Description & UAV A & UAV B \\
\hline$W_{\text {gtow }}$ & Gross take-off weight $[\mathrm{kg}]$ & 10 & 13 \\
\hline$K$ & Lift-drag ratio & 16 & 20 \\
\hline$T / W$ & Thrust-weight ratio & 0.4 & 0.5 \\
\hline$c_{f}$ & Thrust specific fuel consumption $[\mathrm{kg} / \mathrm{kg} \cdot \mathrm{h})]$ & 0.9 & 1.0 \\
\hline$\eta$ & Thrust efficiency & 0.7 & 0.8 \\
\hline$W_{\text {fuel }}$ & Fuel weight $[\mathrm{kg}]$ & 1.0 & 1.2 \\
\hline$V$ & Cruise speed $[\mathrm{km} / \mathrm{h}]$ & 80 & 90 \\
\hline$P_{\text {lim }}$ & Detection probability & 0.7 & 0.6 \\
\hline
\end{tabular}

From the view of UAV performance, $\mathrm{B}$ is superior to $\mathrm{A}$ as Table 2 shows. However, the simulation data show some different results, see Fig. 5. It can be seen from Fig. 5 (a) and (c) that the mean $D_{m c}$ of A is 0.681 , higher than B of which $D_{m c}$ is only 0.605 . It is because a slow speed can lead to a more accurate research. But the deviation of $\mathrm{A}$ is larger than $\mathrm{B}$, because the low $K, W_{\text {fuel }}$ and $\eta$ will lead to a shorter range and endurance. Thus it is easier for A to be affected by uncertainty in the mission. But from the number of cases in which $D_{m c}$ is above $0.5,0.6,0.7,0.8$ or 0.9 , A is still larger than B. So from the view of $D_{m c}$, A is superior than B.

From Fig. 5 (b) and (d), it can be seen that there is not much difference in $P_{t u}$, although the cruise speed of UAV B is higher than UAV A. It is because the time saved by a high cruise speed doesn't play an important role in the whole mission. According to above analysis, A is superior to B. Mission effectiveness analysis based on SoS simulations has an important reference for design evaluation or making mission decisions.

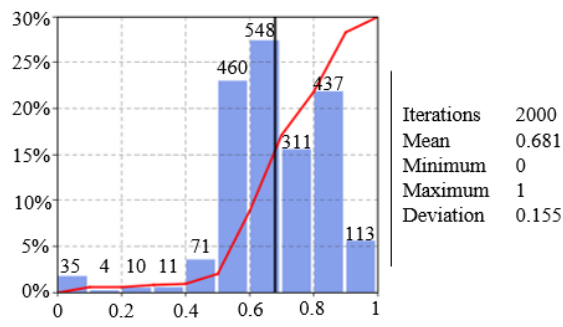

(a). Mission completeness of UAV A.

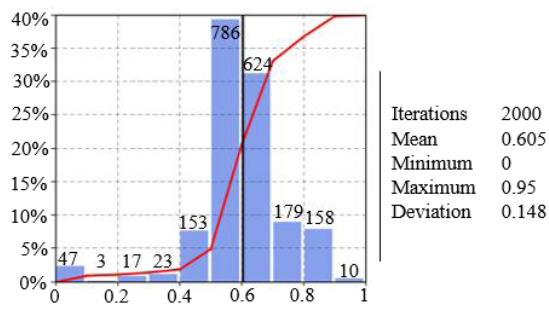

(c). Mission completeness of UAV B.

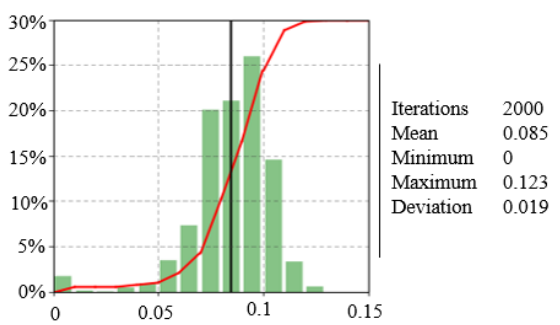

(b). Time utilization ratio of UAV A.

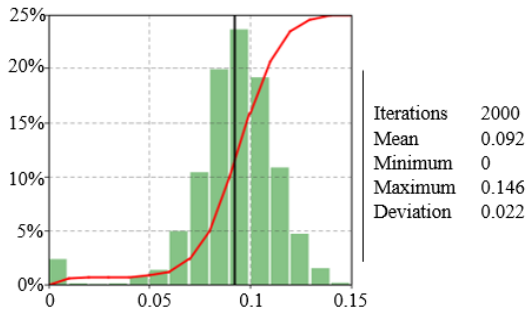

(d). Time utilization ratio of UAV B.

Figure. 5. SoS effectiveness of two UAVs. 


\section{Conclusion}

From the SoS example, it can be seen that SoS simulations provide a feasible way to connect aircraft performance and mission effectiveness. It helps designers to analyse aircraft performance, optimize design parameters and compare different performance designs from the perspective of SoS effectiveness. The results show if designers want to obtain an overall optimum, it may be unnecessary to design aircraft with maximum performance. Through mission simulations, designers could find a more suitable design to save life-cycle time and cost. However, this method still needs more research, for example, more design parameters should be added to simulations as design process continues and design cost should also be taken into account.

\section{References}

1. D. Gorissen, E. Quaranta, M. Ferraro, et al, J. AIRCRAFT 51 5, 1360 (2014)

2. E.S.K. Yu, Third IEEE International Symposium on Requirements Engineering (1997)

3. B. Schumann, J. Scanlan, K. Takeda, Proceedings of the 3rd International Conference on Advances in System Simulation (2011)

4. K.E. Boulding, MANAGE. SCI. 2 3, 197 (1956)

5. A. Gorod, B. Sauser, J. Boardman, IEEE SYST. J. 2 4, 484 (2008)

6. P.T. Biltgen, D.N. Mavris, 45th AIAA Aerospace Sciences Meeting and Exhibit (2007)

7. D.N. Talley, D.N. Mavris, 49th AIAA/ASME/ASCE/AHS/ASC Structures, Structural Dynamics, and Materials Conference (2008)

8. M. Mane, W.A. Crossley, A. Nusawardhana, J. AIRCRAFT 44 4, 1222 (2007)

9. D. DeLaurentis, S. Ayyalasomayajula, J. IND. ECOL. 13 2, 247 (2009)

10. P. Ranque, D. Freeman, K. Kernstine, D. Lim, E. Garcia, D. Mavris, 11th AIAA Aviation Technology, Integration, and Operations (ATIO) Conference (2011)

11. M.W. Maier, SYSTEMS ENG. 1 4, 267 (1998)

12. B. Schumann, J. Scanlan, K. Takeda, 2nd International Air Transport and Operations Symposium (2011) 